\title{
Visualidades expandidas y narrativas transmediales: Derivas de las artes, los lenguajes y los medios Proyecto de Investigación $\mathrm{N}^{\circ} 12.3$
}

Equipo de Investigación ${ }^{(1)}$ Laura Vazquez

por Facultad de Diseño y Comunicación, Universidad de Palermo y el Instituto de Investigaciones Gino Germani, Facultad de Ciencias Sociales, Universidad de Buenos Aires, Conicet (ARG) Maximiliano de la Puente por ICSE-Universidad Nacional de Tierra del Fuego UNTDF, Conicet (ARG)

Resumen: El Proyecto de Investigación 12.3 se propone reflexionar desde y a partir de las artes plásticas, visuales y videográficas contemporáneas, y además desde otras prácticas relacionadas, que dialogan o entran en controversia con los regímenes de representación propios de la modernidad, en tanto esferas autónomas y separadas. Son textos que entablan conversaciones con distintas perspectivas estéticas y que hacen hincapié en diversas gramáticas y regímenes escópicos. Lo que tienen en común es que especulan, cómo las visualidades (plásticas, audiovisuales, gráficas), se expanden a través de las nuevas pantallas y espacios modificando ontológicamente lo que entendemos por artes y narrativas visuales, cinematográficas o de cualquier otro tipo.

Palabras clave: Narrativas contemporáneas - Artes gráficas - Lenguaje - Imagen - Medios de comunicación.

[Resúmenes en inglés y portugués en la página 130]

${ }^{(1)}$ Los CVs del Equipo de Investigación pueden consultarse en el Capítulo Directores de Líneas y Coordinadores de Proyectos de esta misma Edición. 


\section{Acerca del Proyecto 12.3}

\section{Visualidades expandidas y narrativas transmediales: Derivas de las artes, los lenguajes y los medios}

El Proyecto 12.3 Visualidades expandidas y narrativas transmediales: Derivas de las artes, los lenguajes y los medios, analiza el relato visual como el primer eslabón de otro que se relaciona con un concepto ampliado relacionado con la transmedialidad, la cuestión de las marginalidades en el arte, y las artes interaccionando con los medios, para pensar desde distintos prismas teóricos y metodológicos, estos fenómenos críticos de la cultura visual contemporánea. Se reflexiona sobre cómo las visualidades (plásticas, audiovisuales y gráficas) se expanden a través de las nuevas pantallas y espacios, modificando lo que se entiende por narrativas visuales, cinematográficas o de cualquier otro tipo y se hace además, sobre este trasfondo de disparidades y desafíos.

Se acordó entre la Universidad de Palermo, el Instituto de Investigaciones Gino Germani de la Facultad de Ciencias Sociales (UBA. Argentina) y el ICSE-Universidad Nacional de Tierra del Fuego UNTDF (Conicet, ARG), avanzar en una investigación en la que participan académicos de estas Instituciones, con la coordinación compartida de Laura Vazquez (UP e IIGG, UBA) y Maximiliano de la Puente (ICSE, UNTDF Conicet, Argentina), con el fin de generar un corpus sólido de conocimientos y reflexiones a partir de las artes plásticas, visuales y videográficas contemporáneas y sus prácticas relacionadas. Estudia cómo se expanden a través de las nuevas pantallas y espacios modificando ontológicamente lo que entendemos por artes y narrativas visuales, cinematográficas o de cualquier otro tipo. Aborda también las mutaciones que han transformado a las narrativas expandidas visuales, plásticas y gráficas contemporáneas, además de hacer hincapié en los deslindes de las artes, los lenguajes y los géneros. En estas se revisa como se vuelve aglutinante el rol de la autoría teniendo en cuenta las condiciones culturales, políticas, económicas o tecnológicas en las que las obras y producciones tienen o tuvieron lugar.

Las reflexiones y los resultados obtenidos en el Proyecto 12.3 Visualidades expandidas y narrativas transmediales: Derivas de las artes, los lenguajes y los medios, son continuación del Proyecto 12.2 Repensar la alfabetización a partir de la multimodalidad y Proyecto 12.1. Narrativas Gráficas, en el marco de la misma Línea de Investigación y bajo la misma Directora Laura Vazquez.

Sus principales objetivos son:

- Estudiar el modo de creación y producción de los artefactos culturales presentes en la actualidad y las perspectivas teóricas que los sostienen y sistematizan, analizando la novedad y divergencias que circulan en sus soportes mediáticos y en la cultura.

- Indagar la dimensión geográfica en el criterio analítico para atender a las tensiones, las sincronías-asincronías, y las múltiples temporalidades que merecen un abordaje crítico y enfático en términos metodológicos. 
- Poner a prueba las definiciones y cánones históricos de la modernidad en tanto representación para conocer sus espacios, formas de reconocimiento, códigos y modos de circulación.

\section{Acerca de la Línea 12}

Artes Dibujadas dirigida por Laura Vazquez se desarrolla de manera ininterrumpida desde 2015 en la Facultad de Diseño y Comunicación (UP, Argentina), e incluye hasta el momento tres proyectos finalizados el 12.1. Narrativas Gráficas coordinado por Laura Vazquez (UP e IIGG UBA, Conicet, Argentina) e Yvonne Dorelly Quinche Puentes y Jozefh Fernando Soares Queiróz (UP, Argentina), el 12.2 Repensar la alfabetización a partir de la multimodalidad coordinado por Laura Vazquez (UP e IIGG UBA, Conicet, Argentina), Ana Pedrazzini y Nora Scheuer (IIGG UBA, y el IPEHCS, Universidad Nacional del Comahue, Argentina) y el 12.3 Visualidades expandidas y narrativas transmediales: derivas de las artes, los lenguajes y los medios, coordinado por Laura Vazquez (UP e IIGG UBA, Conicet, Argentina) y Maximiliano de la Puente (ICSE-Universidad Nacional de Tierra del Fuego UNTDF, Conicet, Argentina).

\section{Mapa de Áreas y Proyectos}

El Proyecto 12.3 se vincula con todas las carreras de grado y posgrado correspondientes a la Facultad de Diseño y Comunicación, específicamente las relacionadas con el Diseño de Historietas e Ilustración.

\section{Productos y Resultados}

\section{a)- Publicaciones}

Cuaderno del Centro de Estudios de Diseño y Comunicación No119. (2020-2021) Visualidades expandidas y narrativas transmediales: derivas de las artes, los lenguajes y los medios. Coordinación: Laura Vazquez y Maximiliano de la Puente junto al Instituto de Investigaciones Gino Germani-Facultad de Ciencias Sociales UBA, el Instituto de Cultura, Sociedad y Estado- ICSE de la Universidad Nacional de Tierra del Fuego-UNTDF, el Área Transdepartamental de las Artes-Universidad Nacional de las Artes, la Universidad Nacional del Tres de Febrero-UNTREF, Argentina. Facultad de Diseño y Comunicación Año XXIII, Buenos Aires, Argentina. ISSN: 1668-0227.

Esta publicación documenta y comunica los resultados alcanzados en el proyecto de investigación 12.3 Visualidades expandidas y narrativas transmediales: derivas de las artes, los lenguajes y los medios y a continuación se detallan los autores y artículos contenidos en ella: de la Puente Maximiliano, Vazquez Laura (2020-2021) Prólogo. Visualidades expandidas y narrativas transmediales: derivas de las artes, los lenguajes y los medios (Pp.11 a 16) 
García Serventi, Agustín Adolfo (2020-2021) Abordajes teórico críticos en la instalación audiovisual. La teoría del tiempo libre y una estética desde la perspectiva del diseño (Pp. 17 a 30)

Tisera, Martín (2020-2021) Steampunk: análisis del carácter crítico de sus producciones objetuales (Pp. 31 a 45)

Garay Basualdo, Eugenia (2020-2021) El siglo XXI: la curaduría como el boom del momento y los libros específicos (Pp. 47 a 63)

Bortolazzo Pinto, Fabio (2020-2021) Um par de olhos do outro lado do Rio da Prata Como uma historieta expandida de Héctor Oesterheld proporcionou o encontro de um pesquisador brasileiro (Pp. 75 a 88)

Cort, Carolina (2020-2021) De la producción de experiencias a la exhibición de arquitectura (1957-1970). Situacionistas, utopistas y posmodernistas (Pp. 89 a 103) Abadía, Iván (2020-2021) 'Vamo a calmarno'. Los memes como dispositivos de referencialidad comunicativa (Pp. 105 a 132)

Rosso, Aluminé (2020-2021) \#ArteContemporáneo: La arquitectura del museo de arte moderno como discursividad intermediaria. Un análisis de la promesa experiencial (Pp. 133 a 150)

Díaz Quiroga, Lorena (2020-2021) Museo de la inmortalidad. Apuntes para un ensayo transmedia (Pp. 151 a 161)

González, María Laura (Malala) (2020-2021) Arte público contemporáneo y la puerta bicentenaria del GAC ${ }_{¿}$ Hasta dónde es posible seguir hablando de monumentos? (Pp. 163 a 179)

Diéguez, Facundo (2020-2021) Plataformas mediáticas de los museos de artes visuales: mediación técnica, mediatización e interdisciplina. Consideraciones preliminares (Pp. 181 a 189)

La Rocca, Paula (2020-2021) Espacialidad en el conceptualismo latinoamericano. El caso Mirtha Dermisache (Pp. 191 a 207)

Amay, Chantal Arduini (2020-2021) Ficción transmedia en Argentina. El caso de la serie Según Roxi (Pp. 209 a 223)

Bastida Kullick, Edén (2020-2021) Las proyecciones públicas de Krzysztof Wodiczko: arte público, monumento y victimización (Pp. 225 a 244)

Bastida Kullick Edén, Benhumea Salto Nayeli (2020-2021) Pizzurno Pixelado: entre la danza y el premapping (Pp. 245 a 255)

\section{b)- Congresos / Coloquios / Plenarios}

V Coloquio de Investigación y Desarrollo en Diseño Latino. Universidad de Palermo, 27 de julio de 2020. XI Congreso Latinoamericano de Enseñanza del Diseño. Semana Internacional del Diseño en Palermo.

En la comisión Artes Dibujadas, se presentaron las reflexiones y conclusiones del proyecto 12.3 Visualidades expandidas y narrativas transmediales: derivas de las artes, los lenguajes y los medios (Finalizado) expusieron: Agustín García Serventi, Martín Tissera, Malala González, Carolina Corti, Iván Abadia, Facundo Diéguez, Fabio Bortolazzo Pinto, Eugenia 
Basualdo, Paula La Rocca, Lorena Díaz Quiroga, Aluminé Rosso, Chantal Arduini Amaya, Edén Bastida Kullick, Nayeli Benhumea Salto, Laura Vazquez y Maximiliano de la Puente.

A continuación se detallan las ponencias presentadas en esta Comisión coordinada por la Directora del proyecto Laura Vazquez y Maximiliano de la Puente:

Agustín García Serventi (Argentina)

Abordajes teórico críticos en la instalación audiovisual. La teoría del tiempo libre y una estética desde la perspectiva del diseño

Martín Tissera (Argentina)

Steampunk: análisis del carácter crítico de sus producciones objetuales

Malala González (Argentina)

Arte público contemporáneo y la puerta bicentenaria del GAC ¿Hasta dónde es posible seguir hablando de monumentos?

Carolina Corti (Argentina)

De la representación teatral a la exhibición de arquitectura (1957-1970). Situacionismo, utopismo y posmodernismo

Iván Abadia (Argentina)

Vamo a calmarno, los memes como dispositivos de referencialidad comunicativa

Facundo Diéguez (Argentina)

Plataformas mediáticas de los museos de artes visuales: mediación técnica, mediatización e interdisciplina

Fabio Bortolazzo Pinto (Argentina)

Una historieta expandida o como El Eternauta me apresentou à pátria grande

Eugenia Basualdo (Argentina)

El siglo XXI: la curaduría como el boom del momento y los libros específicos

Paula La Rocca (Argentina)

Espacialidad en el conceptualismo latinoamericano. El caso Mirtha Dermisache

Lorena Díaz Quiroga (Argentina)

Museo de la inmortalidad. Apuntes para un ensayo transmedia

Aluminé Rosso (Argentina)

\#ArteContemporáneo: La arquitectura del museo de arte moderno como discursividad intermediaria. Un análisis de la promesa experiencial configurada por los edificios de Centro Pompidou, Malba, Moma y Tate Modern

Chantal Arduini Amaya (Argentina)

Ficción transmedia en Argentina. El caso de la serie Según Roxi

Edén Bastida Kullick (Argentina)

Las proyecciones públicas de Krzysztof Wodiczko: arte público, monumento y victimización

Nayeli Benhumea Salto y Edén Bastida Kullick (Argentina)

Pizzurno Pixelado: entre la danza y el premapping 


\title{
c)- Formación de Posgrado e Impacto curricular
}

La Directora Laura Vazquez es Docente de Posgrado en Diseño de la Facultad de Diseño y Comunicación en Maestría en Gestión del Diseño y Doctorado en Diseño y al igual que Maximiliano de la Puente en su Institución (ICSE-Universidad Nacional de Tierra del Fuego UNTDF, Conicet, Argentina) incorpora los contenidos de su investigación a sus asignaturas en posgrado.

\begin{abstract}
Research Project 12.3 proposes to reflect from and from contemporary plastic, visual and videographic arts, and also from other related practices, which dialogue or enter into controversy with the representation regimes typical of modernity, as autonomous and separate spheres. They are texts that engage in conversations with different aesthetic perspectives and that emphasize various grammars and scopic regimes. What they have in common is that they speculate how visualities (plastic, audiovisual, graphic) expand through new screens and spaces, ontologically modifying what we understand by visual arts and narratives, cinematographic or any other type.
\end{abstract}

Keywords: Contemporary narratives - Graphic arts - Language - Image - Media.

Resumo: O Projeto de Pesquisa 12.3 se propõe a refletir a partir e a partir das artes plásticas, visuais e videográficas contemporâneas, e também de outras práticas afins, que dialogam ou entram em polêmica com os regimes de representação típicos da modernidade, como esferas autônomas e separadas. São textos que conversam com diferentes perspectivas estéticas e que enfatizam várias gramáticas e regimes escópicos. O que eles têm em comum é que especulam como as visualidades (plásticas, audiovisuais, gráficas) se expandem por meio de novas telas e espaços, modificando ontologicamente o que entendemos por artes visuais e narrativas, cinematográficas ou de qualquer outra natureza.

Palavras chave: Narrativas contemporâneas - Artes gráficas - Linguagem - Imagem - Mídia.

[Las traducciones de los resúmenes fueron realizadas a través de traductor automático] 